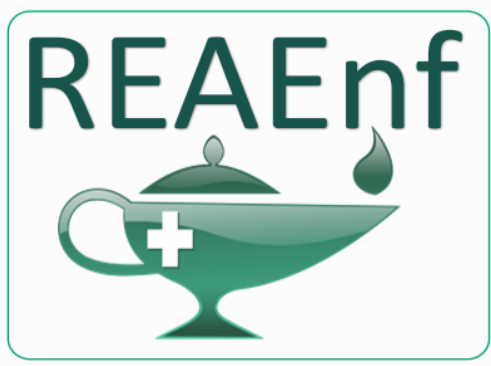

Revista Eletrônica Acervo Enfermagem

\section{REVISÃO BIBLIOGRÁFICA}

Recebido em: $12 / 2019$

Aceito em: 1/2020

Publicado em: 2/2020

\title{
Cobertura vacinal ANTI-HPV e motivos de não vacinação
}

\author{
HPV vacinal coverage and non vaccination reasons
}

\section{Cobertura de vacunación contra PVH y razones de no vacunación}

Rafaela Cristina Alves Altino Almeida ${ }^{1 *}$, Jonathan Mendes de Castro ${ }^{1}$, Talles Vinícius de Castro Oliveira1, Thales Francisco de Oliveira ${ }^{1}$, Diego Azevedo Araújo ${ }^{1}$, Nara Pereira de Faria Carvalho de Alencar ${ }^{1}$, Marcela Alves Azevedo ${ }^{1}$, Jacqueline Souza Dutra Arruda ${ }^{1}$, Carlos Henrique Wernersbach Guerra ${ }^{1}$, Wendel Jose Teixeira Costa ${ }^{1,2}$.

Resumo: O presente estudo teve por objetivo buscar, reunir e sistematizar resultados de investigações científicas nacionais e internacionais, relacionadas à cobertura vacinal contra o Papiloma Virus Humano (HPV) e os motivos de recusa à vacinação. Trata-se de uma revisão integrativa da literatura sobre a cobertura vacinal anti HPV e os motivos de recusa à vacinação. A coleta de dados ocorreu em outubro de 2019 e foram utilizadas na seleção dos artigos, as bases de dados eletrônicos Literatura Latino-Americana em Ciências da Saúde (LILACS) e US National Librare of Medicine (PubMed). Empregaram-se os Descritores em Ciências da Saúde (DeCS) Vacina, Papilomavírus Humano, Recusa de Vacinação e Cobertura de Vacinação no idioma inglês, sem recorte temporal. A literatura analisada demonstrou que a cobertura vacinal anti HPV encontra-se abaixo dos níveis recomendados na literatura e que os principais motivos de recusa vacinal estão relacionados à falta de informação sobre a vacina, principalmente sobre a eficácia e efeitos colaterais e dificuldades de acesso a vacinação, reiterando a necessidade de políticas públicas de educação em saúde, com vistas a informar a população sobre os benefícios da vacina, aumentando assim a adesão por parte da população alvo.

Palavras-chave: Vacina, Papilomavírus humano, Recusa de vacinação

Abstract: The present study aimed to seek, gather and systematize results of national and international scientific investigations related to vaccination coverage against Human Papilloma Virus (HPV) and the reasons for refusal of vaccination. This is an integrative literature review on anti-HPV vaccination coverage and reasons for refusal of vaccination. Data collection took place in October 2019 and were used in the selection of articles, the electronic databases Latin American Literature in Health Sciences (LILACS) and US National Librare of Medicine (PubMed). The Descriptors in Health Sciences (DeCS) Vaccine, Human Papillomavirus, Refusal of Vaccination and Vaccination Coverage in English language were used, without temporal cut. The literature reviewed has shown that anti-HPV vaccine coverage is below the recommended levels in the literature and that the main reasons for vaccine refusal are related to lack of information about

${ }^{1}$ Centro Universitário de Caratinga (UNEC), Caratinga - MG.

2Laboratório de Escrita Científica, Escola Superior de Ciências da Santa Casa de Misericórdia (EMESCAM), Vitória - ES. *E-mail: rafaelaltino2009@hotmail.com 
the vaccine, especially about efficacy and side effects and difficulties in accessing vaccination, reiterating the need for public health education policies to inform the population about the benefits of the vaccine, thereby increasing adherence by the target population.

Keywords: Vaccine, Human papillomavirus, Refusal of vaccination.

Resumen: El presente estudio tuvo como objetivo buscar, recopilar y sistematizar los resultados de las investigaciones científicas nacionales e internacionales relacionadas con la cobertura de vacunación contra el papilomavírus humano (PVH) y los motivos de rechazo de la vacunación. Esta es una revisión bibliográfica integradora sobre la cobertura de vacunación contra el PVH y las razones para rechazar la vacunación. La recopilación de datos tuvo lugar en octubre de 2019 y se utilizó en la selección de artículos, las bases de datos electrónicas eletrônicos Literatura Latino-Americana em Ciências da Saúde (LILACS) e US National Librare of Medicine (PubMed). Se utilizó los Descriptores en Ciencias de la Salud (DeCS): Vacuna, papilomavírus humano, rechazo de vacunación y cobertura de vacunación en inglés, sin límite temporal. La literatura revisada mostró que la cobertura de vacunación contra el PVH está por debajo de los niveles recomendados en la literatura y que las principales razones para el rechazo de la vacuna están relacionadas con la falta de información sobre la vacuna, especialmente sobre la eficacia y los efectos secundarios y las dificultades para acceder a la vacuna. reiterando la necesidad de políticas de educación en salud pública para informar a la población sobre los beneficios de la vacuna, aumentando así la adhesión de la población.

Palabras clave: Vacuna, Papilomavírus humano, Rechazo de la vacunación.

\section{INTRODUÇÃO}

A infecção pelo papiloma vírus humano (HPV) é considerada a doença sexualmente transmissível com maior prevalência mundial, estando associada diretamente ao câncer de colo uterino (MAGl JC, et al., 2006). O HPV é transmitido principalmente por contato sexual, e está associado ao câncer de colo uterino, sendo um relevante problema de saúde pública (BRASIL, 2009).

Em muito dos casos não aparecem sintomas, mas a mulher pode sentir leve prurido, dor na relação sexual e apresentar corrimento vaginal. O Papiloma Vírus Humano pode ficar instalado no corpo por muito tempo sem se manifestar, ocorrendo em casos de imunodepressão fisiológica (GIRIANELLI VR, 2004).

A história natural do HPV e da carcinogênese cervical pode ser representada por um esquema causal composto por quatro estágios: aquisição do HPV, persistência do HPV, progressão para lesão préneoplásica e câncer. Epidemiologicamente, quanto mais tempo uma infecção persistir, maior o risco de desenvolvimento de alterações celulares pré-cancerosas no epitélio e de desenvolvimento de malignidade franca. Em uma duração média desconhecida, a persistência do HPV provavelmente se torna sinônimo de pré-câncer cervical, embora o último possa não ser detectado e até regredir (SCHIFFMAN M, et al., 2007).

A infecção pelo HPV é muito comum na população com vida sexual ativa, porém um câncer ter sua origem a partir de uma infecção é um tanto quanto incomum. Por volta de $90 \%$ das infecções por HPV são benignas e é eliminada ou controlada em 2 anos de tratamento, porém há evidências da possibilidade de algumas infecções se tornarem latentes, estando diretamente relacionadas as alterações celulares e desenvolvimento do pré-câncer cervical (ROSITCH AF, et al., 2012).

O HPV apresenta-se em mais de 100 subtipos, com diferentes potenciais patogênicos e sítios de infecção, alguns deles estão classificados como de alto risco oncogênico por estarem associados a neoplasias malignas do trato genital e não genital enquanto os outros estão vinculados a verrugas genitais e cutâneas (ANDRADE CJ, 2010). Pelo menos 13 deles são considerados oncogênicos. O HPV16 e o HPV18 causam aproximadamente $70 \%$ dos cânceres do colo uterino. Os HPVs mais encontrados nos condilomas genitais e papilomas laríngeos, cerca de 90\%, são considerados não oncogênicos. O HPV16 causa cerca 
de 55 a $60 \%$ dos casos de câncer do colo do útero e o HPV18 cerca de 10 a 15\%. Embora existam variações do genótipo, geralmente estão associados aos mesmos em todo o mundo, com pouca variação regional (DE SANJOSE S, et al., 2010).

É importante ressaltar que, embora a prevalência de genótipos específicos de HPV detectados no tecido tumoral do câncer do colo do útero varie um pouco, geralmente os mesmos genótipos do HPV causam câncer do colo do útero em todo o mundo, com muito pouca variação em sua classificação resumida da importância de região para região (DE SANJOSE S, et al., 2010). Além disso, não há evidências de uma predisposição genética significativa para o câncer cervical, sendo o HPV considerado como principal fator de risco, estando relacionado à quantidade e ao momento da exposição à infecção pelo vírus (LUMMER $H$, et al., 2012), à probabilidade de persistência por infecção e ao acesso a serviços preventivos (MAUCORT BOULCH D, et al., 2008).

Em relação ao câncer de colo uterino ainda é um grande problema de saúde pública, apesar dos programas de detecção precoce baseado no exame Papanicolau (ATHUIS MD, et al., 2005). Representa mais de $95 \%$ dos casos de cânceres, sendo o mais comum entre mulheres no mundo. No Brasil representa a terceira maior causa de mortes entre as mulheres, com aproximadamente, 230 mil óbitos anuais e cerca de 471 mil casos novos (BORSATTO AZ, et al., 2011).

A descoberta do HPV como causa necessária para o desenvolvimento do câncer do colo do útero levou a desenvolvimento de estratégias preventivas, incluindo a vacinação. A vacina contra o HPV demonstrou alto grau de eficácia contra a infecção, e boa relação custo-efetividade, principalmente na prevenção de infecções em adolescentes e jovens com menor probabilidade de exposição pregressa (CASTLE PE e MAZA M, 2015).

Em 2006 a Agência Nacional de Vigilância Sanitária - ANVISA regulamentou a comercialização da vacina que previne contra infecções provocadas por alguns tipos do vírus HPV. A vacina disponível pelo Programa Nacional de Imunização do Ministério da Saúde, é a quadrivalente, oferecendo imunidade contra os subtipos 6,11,16,18, prevenindo contra o câncer cervical (colo uterino), vulvar, anal além da verruga genital, tendo como publico alvo meninas entre 9 à 13 anos e meninos entre 11 à 14 anos, estando disponível também para pessoas de 9 a 26 anos com HIV, em transplantados, pessoas em tratamento oncológico, e em meninas e meninos que chegaram a idade de 15 anos sem completar o esquema vacinal (BRASIL, 2014).

Pesquisas internacionais têm demonstrado que os motivos de recusa à vacina são diversos. Se destacando a necessidade de maiores informações e esclarecimentos sobre da vacina, receio de possíveis eventos adversos, dúvidas sobre a eficácia da vacina, possível estímulo à iniciação sexual precoce e crença de que o exame citopatológico cérvico uterino seja eficiente e suficiente na prevenção do HPV (TOFFOLON-WEISS M, et al., 2008; LAMONTAGNE DS, et al., 2011).

Parte dessa recusa, está relacionada ao movimento anti-vacina, que é constituído por pessoas e pais que não se vacinam e não vacinam seus filhos, afirmam que as vacinas não são eficazes, não são seguras, e consideram as vacinas como uma medida de controle populacional utilizado pelos governos. Essas conspirações circulam o mundo todo, podendo causar sérios danos a saúde da população (OMS, 2019). Com base neste contexto, este estudo tem como objetivo reunir e sistematizar resultados de investigações científicas relacionadas à cobertura vacinal contra HPV e os motivos de recusa à vacinação.

\section{MÉTODOS}

Trata-se de uma revisão integrativa da literatura sobre a cobertura vacinal anti HPV e os motivos de recusa à vacinação. Para o desenvolvimento da presente revisão foram percorridas as seguintes etapas: elaboração da questão norteadora; busca na literatura, seleção dos artigos e coleta de dados; análise crítica dos estudos incluídos; discussão dos resultados, e apresentação da revisão integrativa. A pesquisa partiu da seguinte questão norteadora: Por que a cobertura vacinal anti HPV é baixa? 
A coleta de dados ocorreu em outubro de 2019 e foram utilizadas na seleção dos artigos, as seguintes bases de dados eletrônicos: Literatura Latino-Americana em Ciências da Saúde (LILACS) e US National Librare of Medicine (PubMed).

Empregaram-se os Descritores em Ciências da Saúde (DeCS): Vacina contra Papilomavírus Humano, Recusa de Vacinação e Cobertura de Vacinação no idioma inglês (Human Papillomavirus Vacine AND Vaccination Refusal AND Vaccination Coverage, não foi utilizado recorte temporal. A partir da combinação desses descritores, por meio do operador booleano (AND), foi possível a captura de 22 artigos. No (Quadro 1) encontra-se o roteiro de busca utilizado para a composição da amostra.

Quadro 1 - Distribuição dos estudos capturados segundo combinação dos descritores

\begin{tabular}{|l|c|c|}
\hline Descritores Combinados & $\begin{array}{c}\text { Estudos encontrados em } \\
\text { LILACS }\end{array}$ & $\begin{array}{c}\text { Estudos encontrados em } \\
\text { PubMed }\end{array}$ \\
\hline $\begin{array}{l}\text { Human Papillomavirus Vacine } \\
\text { AND Vaccination Refusal AND } \\
\text { Vaccination Coverage }\end{array}$ & 1 & 21 \\
\hline
\end{tabular}

Fonte: Almeida RCAA, et al., 2019.

Foi realizada a leitura dos títulos e resumos dos 22 artigos capturados nas bases de dados para verificar quais aqueles que respondiam nossa pergunta de pesquisa e se enquadrariam nos critérios de inclusão. Para seleção dos artigos foram estabelecidos critérios de inclusão e exclusão. Priorizou-se a inclusão de artigos disponíveis na íntegra e que respondessem à pergunta proposta por esta revisão. Foram excluídas teses e dissertação. Para a avaliação e apresentação dos artigos incluídos na revisão, foi proposta a construção de uma tabela comparativa enfocando as principais informações levantadas.

\section{RESULTADOS}

A amostra final foi constituída por 6 artigos, sendo cinco artigos da PubMed e um da LILACS, o que demonstra baixa produção científica relacionada a essa temática com média inferior a um estudo por ano, considerando o período de publicação dos artigos incluídos. As temáticas centrais foram cobertura vacinal, estratégias de aumento de cobertura e motivos de recusa vacinal. No (Quadro 2) encontra-se os artigos revisados, assim como sua procedência, título, autores, periódico (volume, número, página e ano) e considerações, temática e resultados.

Os métodos de pesquisa mais frequentes, observados na amostra pesquisada, foram estudos epidemiológicos e análises qualitativas.

No que tange à autoria dos estudos, foi identificado à participação profissionais de saúde ligados a programas governamentais de vacinação e a programas de pós-graduação, docentes, estudantes de graduação e pós-graduação. 
Quadro 2 - Artigos levantados nas bases de dados LILACS e PubMed.

\begin{tabular}{|c|c|c|c|c|}
\hline Procedência & Título do artigo & Autores & $\begin{array}{c}\text { Periódico (vol., } \\
\text { n }^{\circ} \text {, pág., ano) }\end{array}$ & Considerações / Temática/ resultados \\
\hline PubMed & $\begin{array}{l}\text { Diferenças de status } \\
\text { socioeconômico nas } \\
\text { atitudes de } \\
\text { imunização dos pais } \\
\text { e imunização infantil } \\
\text { no Canadá: } \\
\text { resultados da } \\
\text { Pesquisa Nacional } \\
\text { de Cobertura de } \\
\text { Imunizações na } \\
\text { Infância de } 2013 \\
\text { (CNICS). }\end{array}$ & $\begin{array}{l}\text { CARPIAN } \\
\text { O RM, et } \\
\text { al. }\end{array}$ & $\begin{array}{l}\text { Prev Med. } 2019 \\
\text { Jun;123:278-287. }\end{array}$ & $\begin{array}{l}\text { Os esforços de vacinação infantil no Canadá foram impactados negativamente pela hesitação } \\
\text { dos pais com base em seus conhecimentos, atitudes e crenças (KAB) sobre vacinas. Menos } \\
\text { compreendida é a extensão em que o recebimento da vacina infantil e o KAB variam de } \\
\text { acordo com o status socioeconômico dos pais (SES). Analisando diferentes faixas etárias de } \\
\text { crianças e vacinas, examinamos até que ponto (a) o SES da família (educação dos pais, } \\
\text { renda familiar) é um determinante do KAB da vacinação dos pais canadenses e do } \\
\text { recebimento da vacinação infantil; e (b) se o SES foi indiretamente associado com recibo via } \\
\text { KAB. }\end{array}$ \\
\hline PubMed & $\begin{array}{l}\text { Fatores associados } \\
\text { à recusa da vacina } \\
\text { contra o HPV entre } \\
\text { mulheres adultas } \\
\text { após dez anos de } \\
\text { implementação da } \\
\text { vacina }\end{array}$ & $\begin{array}{l}\text { RESTIVO } \\
\text { V, et al. }\end{array}$ & $\begin{array}{c}\text { Int J Environ Res } \\
\text { Public Health. } \\
2018 \\
\text { Apr17;15(4):770. }\end{array}$ & $\begin{array}{l}\text { Fatores associados à recusa da vacinação contra o HPV foram o nível de escolaridade (OR = } \\
10,2 \text {, sem iniciar ou concluir a vacinação contra o HPV. No geral, } 141 \text { mulheres jovens foram } \\
\text { matriculadas (taxa de resposta } 22 \% \text {. Entre eles, } 84,4 \% \text { não foram vacinados e } 15,6 \% \text { tinham } \\
\text { pelo menos uma dose da vacina contra o HPV. O programa educacional de saúde pública, } \\
\text { focado e adaptado à percepção dos benefícios da vacina contra o HPV e da gravidade da } \\
\text { doença, realizado na escola ou durante visitas médicas, pode ser útil para melhorar a } \\
\text { aceitação da vacina contra o HPV. }\end{array}$ \\
\hline PubMed & $\begin{array}{c}\text { Pais que recusam a } \\
\text { vacinação contra o } \\
\text { HPV: quem mais } \\
\text { tarde aceita e por } \\
\text { quê? }\end{array}$ & $\begin{array}{l}\text { KORNI } \\
\text { DES } \\
\text { ML, et } \\
\text { al. }\end{array}$ & $\begin{array}{c}\text { AcadPediatr. } \\
2018 \\
\text { Mar;18(2S):S37- } \\
\text { S43 }\end{array}$ & $\begin{array}{l}\text { No geral, } 45 \% \text { dos pais relataram aceitação secundária da vacinação contra o HPV e outros } \\
24 \% \text { pretendem vacinar nos próximos } 12 \text { meses. Nas análises multivariáveis, a aceitação } \\
\text { secundária foi associada ao recebimento de aconselhamento de acompanhamento de um } \\
\text { profissional de saúde sobre a vacinação contra o HPV (oddsratio, } 2,16 \text {; intervalo de confiança } \\
\text { de } 95 \%, 1,42-3,28 \text { ). No entanto, apenas } 53 \% \text { dos pais relataram ter recebido esse } \\
\text { aconselhamento. A aceitação secundária também foi associada ao recebimento de uma } \\
\text { recomendação de alta qualidade da vacina contra o HPV de um fornecedor durante a } \\
\text { discussão inicial e maior satisfação com a comunicação do fornecedor, além de maior } \\
\text { confiança na vacinação. Entre os motivos da aceitação secundária, os pais relataram com } \\
\text { mais frequência que a criança estava ficando mais velha (45\%), aprendendo mais sobre a } \\
\text { vacina contra o HPV (34\%) e recebendo uma recomendação do fornecedor (33\%). }\end{array}$ \\
\hline
\end{tabular}

REAEnf/EJNC | Vol.2 | e2600 | DOI: https://doi.org/10.25248/REAenf.e2600.2020 Página 5 de 9 


\begin{tabular}{|c|c|c|c|c|}
\hline PubMed & $\begin{array}{l}\text { Estratégias de } \\
\text { vacinação para } \\
\text { adolescentes: } \\
\text { intervenções para } \\
\text { aumentar a } \\
\text { cobertura. }\end{array}$ & $\begin{array}{c}\text { LEHMANN } \\
\text { CE, et al. }\end{array}$ & $\begin{array}{c}\text { Paediatr Drugs. } \\
2016 \\
\text { Aug;18(4):273- } \\
285 .\end{array}$ & $\begin{array}{l}\text { Embora as vacinas diminuam o ônus da doença, muitos adolescentes ainda permanecem sub- } \\
\text { imunizados, principalmente para o papilomavírus humano (HPV) e influenza. Analisamos os } \\
\text { dados mais atuais sobre imunizações de adolescentes nos Estados Unidos e discutimos } \\
\text { estratégias comprovadas que funcionam para aumentar as taxas de vacinação. As estratégias } \\
\text { que demonstraram melhorar as taxas incluem feedback do fornecedor, sistemas de } \\
\text { informação sobre imunização (ou registros) e acesso aprimorado fora dos escritórios do } \\
\text { fornecedor, como programas de imunização nas escolas. No geral, as práticas podem querer } \\
\text { considerar abordagens de melhoria da qualidade multimodal para aumentar as taxas de } \\
\text { vacinação na prática. A saúde pública e os benefícios de custo da imunização de } \\
\text { adolescentes são bem conhecidos, no entanto, surtos recentes de sarampo nos Estados } \\
\text { Unidos destacaram questões com leis estaduais de imunização e recusas de vacinas. . }\end{array}$ \\
\hline PubMed & $\begin{array}{c}\text { Estimativas e } \\
\text { determinantes da } \\
\text { não vacinação } \\
\text { contra o HPV e } \\
\text { recusa de vacina em } \\
\text { meninas de } 12 \text { a } 14 \\
\text { anos de idade no } \\
\text { Canadá: Resultados } \\
\text { da Pesquisa } \\
\text { Nacional de } \\
\text { Cobertura de } \\
\text { Imunizações na } \\
\text { Infância, 2013.. }\end{array}$ & $\begin{array}{l}\text { GILBERT } \\
\text { NL, et al. }\end{array}$ & $\begin{array}{c}\text { Hum Vaccin } \\
\text { Immunother. } \\
2016 \\
\text { Jun2;12(6):1484- } \\
1490 . .\end{array}$ & $\begin{array}{l}\text { Os dados da Pesquisa Nacional de Cobertura da Imunização da Infância (CNICS) de } 2013 \\
\text { foram usados para estimar a prevalência de não vacinação contra o HPV e recusa da vacina } \\
\text { dos pais em meninas de } 12 \text { a } 14 \text { anos, no Canadá e nas províncias e territórios. A regressão } \\
\text { logística multivariada foi usada para examinar os fatores associados à não vacinação e recusa } \\
\text { da vacina, após o ajuste para possíveis fatores de confusão. Estima-se que } 27,7 \% \text { das } \\
\text { meninas de } 12 \text { a } 14 \text { anos não haviam sido vacinadas contra o HPV e } 14,4 \% \text { dos pais } \\
\text { relataram ter recusado a vacina. A magnitude da não vacinação e da recusa de vacina variou } \\
\text { de acordo com a província ou território e também com o país de nascimento dos pais em } \\
\text { resposta. Além disso, o ensino superior foi associado a um maior risco de recusa da vacina } \\
\text { contra o HPV. As taxas de não vacinação contra o HPV e de recusa da vacina contra o HPV } \\
\text { diferem e são influenciadas por diferentes variáveis. Esses achados justificam uma } \\
\text { investigação mais aprofundada. }\end{array}$ \\
\hline LILACS & $\begin{array}{l}\text { Estado vacinal e } \\
\text { motivos de não } \\
\text { vacinação contra o } \\
\text { vírus papiloma } \\
\text { humano em } \\
\text { adolescentes } \\
\text { admitidas no } \\
\text { Hospital Pediátrico } \\
\text { do Centro } \\
\text { Hospitalario Pereira } \\
\text { Rossell }\end{array}$ & $\begin{array}{l}\text { NOTEJAN } \\
\text { E M, et al. }\end{array}$ & $\begin{array}{c}\text { Rev. méd. } \\
\text { Urug;34(2): } 76- \\
\text { 81, jun. } 2018 . \\
\text { tab, ilus }\end{array}$ & $\begin{array}{l}\text { motivo de não vacinação mais frequente foi o desconhecimento da existência da vacina } \\
(71,6 \%) \text {, seguido de rejeição ou negativa da adolescente ou do adulto responsável }(19,4 \%) \text {. } 0 \\
\text { motivo principal de rejeição a ser vacinada foi a falta de informação. Não foram registrados } \\
\text { efeitos adversos graves. Conclusões: a cobertura vacinal registrada foi similar aos dados } \\
\text { nacionais. O conhecimento e a informação das adolescentes e de seus cuidadores sobre esta } \\
\text { vacina foram os principais motivos de não vacinação detectados. }\end{array}$ \\
\hline
\end{tabular}

Fonte: Almeida RCAA, et al., 2019.

REAEnf/EJNC | Vol.2 | e2600 | DOI: https://doi.org/10.25248/REAenf.e2600.2020 Página 6 de 9 


\section{DISCUSSÃO}

$\mathrm{Na}$ Itália um estudo demonstrou baixa cobertura vacinal anti HPV e dificuldades de manutenção adequada da cobertura em diversas regiões de saúde. Maior recusa foi associada a níveis mais elevados de escolaridade e entre que não tiveram orientações na escola sobre o HPV. Os principais motivos estavam relacionados à falta de informação sobre eficácia e efeitos colaterais da vacina, dificuldades de acesso e nível superior de escolaridade. Essa falta de informação foi relatada principalmente entre as mulheres jovens, estas que nunca realizaram a vacinação. Pessoas que não tem informações sobre a vacina não sentem a necessidade de realizá-la, diminuindo assim a taxa de cobertura vacinal. $O$ fornecimento de informações, seguras e corretas sobre os benefícios da vacinação e os riscos da doença, incluindo possíveis efeitos colaterais em seus níveis de apresentação, são estratégias fundamentais para a conscientização dessas mulheres com respeito a vacinação anti-HPV (RESTIVO V, et al., 2018).

Achados semelhantes foram encontrados em dois estudos realizados no Canadá, onde a vacinação contra HPV foi impactada negativamente pela hesitação dos pais com base em seus conhecimentos, atitudes e crenças sobre vacinas. Condições sócio-econômicas desfavoráveis como baixa renda familiar e baixo nível de escolaridade dos pais, estiveram associados à menor cobertura vacinal (GILBERT NL, et al., 2016; CARPIANO RM, et al., 2019).

A indecisão vacinal é um fenômeno complexo, contexto-específico, que varia no tempo, nos lugares, com tipos de vacinas, e como estratégia de solução desse problema, se faz necessário monitorar a confiança nas vacinas e os motivos de recusa vacinal, bem como desenvolver intervenções eficazes de comunicação para enfrentar as falhas na confiança em vacinas, evitar suas consequências para a saúde pública (SUCCI RCM, 2018).

No Uruguai, a cobertura vacinal contra HPV foi próxima a 40\%, muito abaixo da média recomendada, e também se comparada com a cobertura de outras vacinas. O conhecimento e a informação das adolescentes e de seus cuidadores sobre a vacina anti HPV, também foram os principais motivos de não vacinação detectados. (NOTEJANE M, et al., 2018). Nos Estados Unidos, dois estudos observaram que a recusa dos pais contribuiu para a baixa cobertura vacinal anti HPV entre adolescentes, resultando em oportunidades perdidas de prevenção ao câncer do colo uterino. Os principais motivos de recusa também estiveram relacionados à falta de informações sobre os reais benefícios da vacina, bem como a crenças de riscos de efeitos colaterais graves (LEHMANN CE, et al., 2016; KORNIDES ML, et al., 2018).

Para aumento da cobertura vacinal contra HPV nos Estados Unidos foram utilizadas estratégias que demonstraram melhorar as taxas de cobertura, como feedback do fornecedor, sistemas de informação sobre imunização e programas de imunização nas escolas, sugerindo que essas estratégias poderiam ser ampliadas e implementadas em regiões com menores taxas (LEHMANN CE, et al., 2016).

Estratégias de educação em saúde, com garantia de informações sobre os riscos da doença, medidas de prevenção, informações seguras sobre os benefícios da vacinação, esclarecendo dúvidas, medos e riscos de eventos adversos graves, são consideradas fundamentais para influenciar a conscientização sobre a vacinação contra o HPV (GIAMBI C, et al., 2014). Percebe-se que é muito importante a promoção da saúde a partir de métodos educativos para melhorar a capacidade de decisão por parte da população alvo e seus familiares, pais ou responsáveis, e conseqüentemente maior adesão a vacina (ATRASH $\mathrm{K} \mathrm{e}$ CARPENTIER, 2012).

A taxa de cobertura vacinal contra o HPV tem sido reportada como abaixo do recomendado em diversos países. Uma revisão sistemática da literatura realizada no ano de 2019 expôs essa condição ao abordar as barreiras à vacinação na América Latina. Segundo esse estudo os fatores se distinguem em duas categorias principais: fatores de acessibilidade e fatores individuais. Ao retratar sobre os fatores de acessibilidade, a falta de infraestrutura e de apoio financeiro adequados são alguns dos elementos que resultam na baixa cobertura, além de citar o não adequado fornecimento de conhecimento e treinamento de profissionais de saúde e da falta de informação sobre as vacinas, tanto pelos profissionais, como pela população em geral (GUZMAN-HOLST A, et al., 2019). 
Ao retratar os fatores individuais foi abordada a aceitação e recusa pela vacina. A recusa parte de diversos fatores, como a falta de confiança e de conhecimento dos benefícios e da necessidade da vacina, além da acessibilidade à vacinação, por vezes muito difícil em alguns países, especialmente em regiões rurais e periféricas. $O$ estudo também destacou que os movimentos anti-vacinas também representaram um fator importante para a recusa à vacinação, pois ao estudar a baixa da cobertura vacinal, no Brasil, por exemplo, houve associação de difusão de falsas informações e, por conseguinte, baixa cobertura vacinal em diversas regiões do país e um subsequente ressurgimento do sarampo em 2018 (SRIDHAR S, et al., 2019).

A hesitação na aceitação da vacina é influenciada por três fatores principais: desconfiança, que é a falta de confiança na vacina ou no fornecedor; complacência, que é a percepção de que não há valor, importância ou necessidade de uma vacina e conveniência, que se refere à falta de acesso ou serviços de vacinação. Influenciados por esses fatores, os indivíduos podem recusar algumas vacinas ou retardar a vacinação (OMS, 2019). Outro motivo importante para vacinação perdida entre crianças e adultos é a falta de conscientização ou informações inadequadas fornecidas pelas autoridades de saúde e profissionais de saúde, o que leva à criação de falsas crenças sobre os benefícios da vacinação e seus efeitos adversos (TOHME RA, et al., 2014).

De acordo com as diretrizes da Política de Saúde Integral do Adolescente e Jovem, os profissionais de saúde devem estar preparados para implementar medidas de promoção da saúde e de prevenção de agravos, incluindo a vacinação, de acordo com o calendário vacinal o que torna essencial adaptações nas relações interpessoais dos profissionais com os adolescentes e seus familiares, tendo o diálogo como fundamento para o cuidado integral. As necessidades de saúde dos adolescentes dependem da qualidade de sua interação biológica, psicológica e social, o que direciona para ações de saúde pública mais eficientes, abrangentes e criativas (COSTA MCO e BIGRAS M, 2017).

No que diz respeito ao conhecimento, destaca-se a importância de os adolescentes terem acesso à informação, que permita avaliar o seu próprio risco de adquirir uma doença imunoprevenível, motivando-os para aceitarem a vacinação (SCHIMIDT M e MIDDLEMAN AB, 2001). Sendo essencial um trabalho intersetorial efetivo incluindo as escolas e professores nas estratégias de vacinação, para melhor aceitação da vacina (TUNG CS e MIDDLEMAN AB, 2005).

Portanto, é importante refletir essa temática da vacinação sob a ótica das relações entre as famílias, comunidade, redes de apoio e os serviços de saúde, a partir da fundamentação teórica acerca do cuidado e reconstrução das práticas de saúde, abordando o cuidado numa perspectiva de interação entre sujeitos, implicando a percepção e a construção da intersubjetividade (AYRES JRCM, 2004).

\section{CONSIDERAÇÕES FINAIS}

A literatura analisada demonstra que a cobertura vacinal anti HPV encontra-se abaixo dos níveis recomendados e que os principais motivos de recusa vacinal estão relacionados à falta de informação sobre a vacina, principalmente sobre a eficácia e efeitos colaterais e dificuldades de acesso a vacinação. Referese então a necessidade da implementação de políticas públicas mais adequadas à situação, tendo a educação em saúde como foco, capacitando os profissionais de saúde e orientando a população em geral quanto à eficácia e efeitos colaterais da vacina.

\section{REFERÊNCIAS}

1. ALTHUIS MD, et al. Global trends in breast cancer incidence and mortality 1993-1997. Int J Epidemiol, 2005; 34(2): 405-12.

2. ANDRADE CJ. Avaliações econômicas do uso da vacina contra o Papilomavírus Humano (HPV) em meninas adolescentes: uma revisão sistemática [dissertação]. Rio de Janeiro: Centro Biomédico Instituto de Medicina Social, Universidade do Estado do Rio de Janeiro; 2010.

3. AYRES JRCM. Cuidado e reconstrução das práticas de saúde. Interface Comun Saúde Educ 2004;8(14):73-92.

REAEnf/EJNC | Vol.2 | e2600 | DOI: https://doi.org/10.25248/REAenf.e2600.2020 Página 8 de 9 
4. BORSATTO AZ, et al. HPV Vaccine and the Prevention of Cervical Uterus Cancer: Subsidies to the Practice, Revista Brasileira de Cancerologia, 2011; 57(1): 67-74.

5. BRASIL. Ministério da Sáude. Instituto Nacional do Câncer. Estimativa 2010: incidência de câncer no Brasil / Instituto Nacional de Câncer. - Rio de Janeiro: INCA, 2009. 98p.

6. BRASIL. Ministério da Saúde. Manual de Normas e Procedimentos para Vacinação / Ministério da Saúde, Secretaria de Vigilância em Saúde, Departamento de Vigilância das Doenças Transmissíveis. - Brasília: Ministério da Saúde, 2014. 176 p.: il.

7. CARPIANO RM, et al. Socioeconomic status differences in parental immunization attitudes and child immunization in Canada: Findings from the 2013 Childhood National Immunization Coverage Survey (CNICS). Preventive Medicine, 2019; 123(2019): 278-287.

8. CASTLE PE, MAZA M. Prophylactic HPV vaccination: past, present, and future. Epidemiology and Infection, 2015; 144(03): 449-468.

9. COSTA MCO, BIGRAS M. Mecanismos pessoais e coletivos de proteção e promoção da qualidade de vida para a infância e adolescência. Ciênc Saúde Coletiva 2007;12(5):1101-109.

10. DE SANJOSE S, et al. Atribuição do genótipo do papilomavírus humano no câncer cervical invasivo: um estudo retrospectivo transversal em todo o mundo. Lancet Oncology, 2010; 11: 1048 - 1056.

11. GIAMBI $C$ et al. Local Representatives for VALORE. Exploring reasons for non-vaccination against human papillomavirus in Italy. BMC Infect. Dis. 2014, 14: 545.

12. GILBERT NL, et al. Estimates and determinants of HPV non-vaccination and vaccine refusal in girls 12 to $14 \mathrm{y}$ of age in Canada: Results from the Childhood National Immunization Coverage Survey, 2013. Human Vaccines \& Immunotherapeutics, 2016; 12(6): 1484-1490.

13. GIRIANELLI VR. Comparison of hybrid capture II, liquid based cytology and Pap test for the early detection of the cervical cancer in Rio de Janeiro, Brazil, Rev. bras. Cancerol, 2004; 50(3): 225-226.

14. GUZMAN-HOLST A, et al. Barriers to vaccination in Latin America: A systematic literature review.

15. KORNIDES ML, et al. Parents Who Decline HPV Vaccination: Who Later AcceptsandWhy? AcadPediatr, 2018; 18(2S): S37-S43.

16. LAMONTAGNE DS, et al. Human papillomavirus vaccine delivery strategies that achieved high coverage in low- and middle-income countries. Bull World Health Organ., 2011; 89(11): 821-830B.

17. LEHMANN CE, et al. Adolescent Vaccination Strategies: Interventions to Increase Coverage. PaediatrDrugs, 2016; 18(4):273-85.

18. LUMMER $\mathrm{H}$, et al. Tempo desde a primeira relação sexual e o risco de câncer do colo do útero. Revista Internacional de Câncer, 2012; 130: 2638 - 2644.

19. MAGI JC, et al. Prevalência de papilomavírus humano (HPV) anal, genital e oral, em ambulatório geral de coloproctologia. RevBras Colo-Proctol, 2006; 3(26): 233-238.

20. MAUCORT-BOULCH D, et al. Correlação internacional entre prevalência de papilomavírus humano e incidência de câncer cervical. Epidemiologia do Câncer, Biomarcadores e Prevenção, 2008; 17: 717 - 720.

21. NOTEJANE M, et al. Vaccination status andreasons for non-vaccination against human papilloma virus (HPV) in adolescents admitted at the Pereira Rossell Hospital Center Pediatrics Hospital. Rev. méd. Urug, 2018; 34(2): 76 81.

22. OMS. Organização Mundial da Saúde (website) \# Ten threats to global health in 2019;

23. RESTIVO V, et al. Factors Associated with HPV Vaccine Refusal among Young Adult Women after Ten Years of Vaccine Implementation. Int J Environ Res Public Health, 2018; 15(4): 770.

24. ROSITCH AF, et al. Contribuições de parcerias sexuais recentes e passadas na detecção incidente de papilomavírus humano: aquisição e reativação em mulheres mais velhas. Pesquisa de Câncer 2012; 72 : 6183 6190.

25. SCHIFFMAN M, et al. Papilomavírus humano e câncer cervical. Lancet, 2007; 370: 890 - 907.

26. SCHIMIDT M, MIDDLEMAN AB. The importance of hepatitis $B$ vaccination among adolescents. J Adolesc Health $2001 ; 29: 217-22$.

27. SRIDHAR S, et al. A systematic literature review of missed opportunities for immunization in low- and middle-income countries. Vaccine, 2014; 32(2014): 6870-6879.

28. SUCCI RCM. Vaccine refusal --- what we need to know. J Pediatr (Rio J). 2018; 94(6): 574-581.

29. TOFFOLON-WEISS M, et al. Alaska Native parental attitudes on cervical cancer, HPV and the HPV vaccine. Int $J$ Circumpolar Health., 2008; 67(4): 363-373.

30. TOHME RA, et al. Measles and rubella vaccination coverage in Haiti, 2012: progress towards verifying and challenges to maintaining measles and rubella elimination. Trop Med Int Health, 2014; 19(2014): 1105-1115. 